\title{
State estimation for anaerobic digesters using the ADM1
}

\author{
D. Gaida, C. Wolf, C. Meyer, A. Stuhlsatz, J. Lippel, T. Bäck, M. Bongards \\ and S. McLoone
}

\begin{abstract}
The optimization of full-scale biogas plant operation is of great importance to make biomass a competitive source of renewable energy. The implementation of innovative control and optimization algorithms, such as Nonlinear Model Predictive Control, requires an online estimation of operating states of biogas plants. This state estimation allows for optimal control and operating decisions according to the actual state of a plant. In this paper such a state estimator is developed using a calibrated simulation model of a full-scale biogas plant, which is based on the Anaerobic Digestion Model No.1. The use of advanced pattern recognition methods shows that model states can be predicted from basic online measurements such as biogas production, $\mathrm{CH}_{4}$ and $\mathrm{CO}_{2}$ content in the biogas, $\mathrm{pH}$ value and substrate feed volume of known substrates. The machine learning methods used are trained and evaluated using synthetic data created with the biogas plant model simulating over a wide range of possible plant operating regions. Results show that the operating state vector of the modelled anaerobic digestion process can be predicted with an overall accuracy of about $90 \%$. This facilitates the application of state-based optimization and control algorithms on full-scale biogas plants and therefore fosters the production of eco-friendly energy from biomass.

Key words | ADM1, anaerobic digestion, GerDA, optimal control, pattern recognition, state estimation

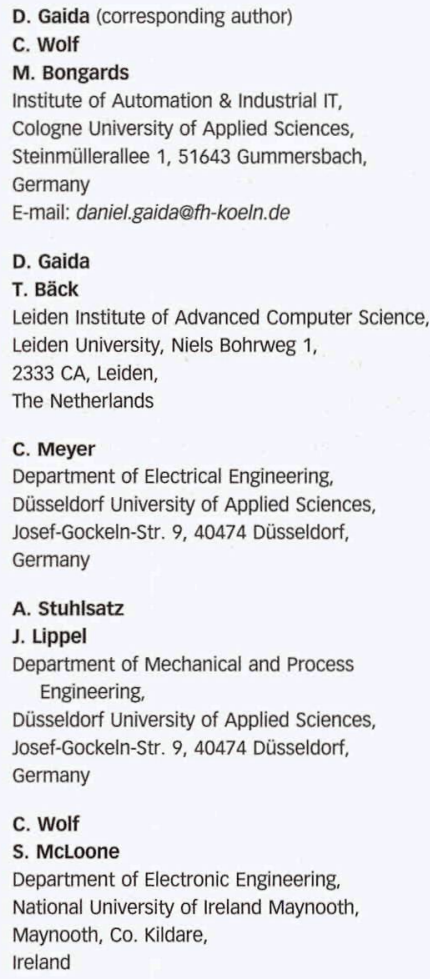

\section{INTRODUCTION}

Anaerobic digestion is a process by which biomass is converted to biogas, which mainly consists of methane $\left(\mathrm{CH}_{4}\right)$, carbon dioxide $\left(\mathrm{CO}_{2}\right)$ and hydrogen $\left(\mathrm{H}_{2}\right)$. In biogas plants this process is used to produce energy by either burning the biogas in cogeneration units and supplying the energy to the national grid or by the processing of biogas to natural gas quality for supply to the local gas distribution system. Despite the high potential of biogas production from biomass in biogas plants, to contribute significantly to the European Renewable Energy Policy (Directive 2009/28/EC of the European Parliament 2009), plant operation is far from optimal in most cases (Schmitz 2004). The reasons are obvious. Not only is anaerobic digestion a highly complex and nonlinear dynamic process but most biogas plants suffer from a lack of robust online-measurement systems for close process monitoring (Wiese \& König 2009). Unfortunately, nonstandard measurement equipment for critical process parameters such as organic acid concentrations and buffer capacity are too expensive and require extensive maintenance and expert knowledge from plant operators. Consequently only basic measurement systems are available on the typical agricultural biogas plant. Typically, just biogas production, biogas composition, $\mathrm{pH}$ value, redox potential, total solids content and temperature sensors are available (Etzkorn 2008). This makes optimal control and optimization of anaerobic digestion processes very difficult and challenging.

As hardware measurement sensors are expensive, an alternative approach is to explore soft-sensing of key process variables (Fortuna et al. 2007). These soft-sensors provide 
predictions of difficult to measure variables from basic (more accessible) process measurements by assuming that a relationship exists between key and basic plant measurements, which can be modelled (Bernard et al. 200I). In this paper such a soft sensor is developed to estimate variables describing the internal state of a biogas plant, thus providing a state estimator for biogas plants.

The developed state estimator can be seen as a static function, which estimates the current internal state of the anaerobic digestion process based on current and past measurement data of a biogas plant. The main advantage of this approach compared with conventional state estimation filters (Rawlings \& Bakshi 2006) is that an initial guess of the initial state of the biogas plant is not necessary. To allow for good practical applicability only those measurement variables are considered, which are commonly measured on most biogas plants. Thus, biogas production, methane and carbon dioxide content in the biogas, $\mathrm{pH}$ value inside the digesters and the amount of each substrate fed to the fermentation tanks are considered in this approach.

Discriminant analysis and classification based pattern recognition methods are used to find the static mapping function, i.e. the pattern, between the measurement data and the internal anaerobic digestion state. As discriminant analysis methods the well-known Linear Discriminant Analysis (LDA) and the newly developed Generalized Discriminant Analysis (GerDA) (Stuhlsatz et al. 2010a) are used to extract optimal classifiable features from the measurements. Classification of the resulting features is then performed using a linear classifier. Furthermore, Random Forest is investigated for classification of the raw measurements without a feature extraction step.

As the internal states of the anaerobic digestion process are not measurable using standard measurement equipment, the data used for training and evaluating the pattern recognition methods under investigation has to be created synthetically. Therefore a detailed simulation model of a full-scale agricultural biogas plant is developed using the widely used Anaerobic Digestion Model No. 1 (ADM1) (Batstone et al. 2002). Thus, the internal state of anaerobic digestion is defined by the ADM1 state vector. In Gaida et al. (20пb) a Nonlinear Model Predictive Control (NMPC) scheme is developed to optimally control fullscale biogas plants. This NMPC method requires an online estimate of the plant's current operating state. Thus, a combination of the proposed state estimator and the NMPC method will make it possible to deploy NMPC in practice and thus facilitate the control of full-scale biogas plants in the near future.
The remainder of the paper is structured as follows. In the next section the biogas plant model, the dataset and the machine learning methods employed are briefly described. Then the results obtained with the proposed state estimation scheme using the various pattern recognition methods are presented and analysed.

\section{$\overline{\text { MATERIALS AND METHODS }}$}

This section describes the real full-scale biogas plant, the dynamic simulation model and the synthetically generated dataset. The pattern recognition methods used to learn the mapping function of the state estimator are also introduced.

\section{The biogas plant}

The biogas plant under consideration is a full-scale agricultural biogas plant with an electrical power output of $750 \mathrm{~kW}$ located in Germany. The plant contains two digesters with a volume of about $3,000 \mathrm{~m}^{3}$ each. Only the first digester is fed with substrates including maize, grass and manure.

To create the synthetic dataset a simulation model is developed and calibrated for this biogas plant. Modelling anaerobic digestion using the complex ADM1 has proven to be a very good and all-round approach (Lübken et al. 2007; Page et al. 2008; Koch et al. 2010). The ADM1 is commonly implemented as a non-linear differential equation system of the form:

$$
\begin{array}{cc}
\mathbf{x}^{\prime}(t)=\mathbf{g}(\mathbf{x}(t), \mathbf{u}(t)), & \text { system function: } \\
& \mathbf{g}: \mathbf{R}^{n} \times \mathbf{R}^{u} \rightarrow \mathbf{R}^{n} \\
\mathbf{y}(t)=\mathbf{h}(\mathbf{x}(t), \mathbf{u}(t)), & \text { measurement function: } \\
\mathbf{h}: \mathbf{R}^{n} \times \mathbf{R}^{u} \rightarrow \mathbf{R}^{m}
\end{array}
$$

Here the, time $t \in \mathbf{R}^{+}$dependent input vector function $\mathbf{u}: \mathbf{R} \rightarrow \mathbf{R}^{u}$ is defined by the volume flows $Q_{\text {substrate }} \in \mathbf{R}$ of the $u=5$ available substrates, which are measured in $\mathrm{m}^{3} / \mathrm{d}$, that is:

$\mathbf{u}=\left[Q_{\text {maize }}, Q_{\text {manure }}, Q_{\text {siloseepage }}, Q_{\text {grass }}, Q_{\text {manuresolids }}\right]^{\mathrm{T}}$

The physical and chemical parameters of the substrates are assumed to be constant, so that the developed estimator only yields valid results for substrate characteristics the estimator has learned during training. The 
output vector function $\mathbf{y}: \mathbf{R} \rightarrow \mathbf{R}^{m}$ is composed of the simulated $\mathrm{pH}$ values inside the two digesters $\left(\mathrm{pH}_{1}, \mathrm{pH}_{2}\right)$, the produced biogas volume flows $\left(Q_{\text {biogas, } 1}, Q_{\text {biogas, } 2}\right)$ and the relative amount of methane and carbon dioxide $\left(V_{\mathrm{CH}_{4}, 1}, V_{\mathrm{CH}_{4}, 2}, V_{\mathrm{CO}_{2}, 1}, V_{\mathrm{CO}_{2}, 1}\right)$ in the produced biogas. Thus, in total there are $m=8$ measurement variables, four for each digester:

$$
\begin{gathered}
\mathbf{y}=\left[\mathrm{pH}_{1}, Q_{\text {biogas }, 1}, V_{\mathrm{CH}_{4}, 1}, V_{\mathrm{CO}_{2}, 1}, \mathrm{pH}_{2}, Q_{\text {biogas }, 2},\right. \\
\left.V_{\mathrm{CH}_{4}, 2}, V_{\mathrm{CO}_{2}, 2}\right]^{\mathrm{T}}
\end{gathered}
$$

It is important to note that output vector function $\mathbf{y}$ and input vector function $\mathbf{u}$ were chosen deliberately so that they contain process parameters, which are measured in practice on almost every biogas plant. The $n=37$ components of the ADM1 state vector $\mathbf{x}: \mathbf{R} \rightarrow \mathbf{R}^{n}$ are shown in Figure 1 in the results section.

The simulation model of the biogas plant is developed in MATLAB ${ }^{\circledR} /$ Simulink (Gaida et al. 20па). The ADM1 parameters are set to standard values, except for the parameters $K_{\mathrm{S}, \mathrm{c} 4}, k_{m, \mathrm{pro}}, k_{m, \mathrm{ac},} K_{\mathrm{I}, \mathrm{NH} 3}$ and $k_{m, \mathrm{~h} 2}$, as recommended in Wichern et al. (2007, 2009).

The characterization of the ADM1 input stream is based on the following measurements, which have been performed for all substrates. In laboratory analysis, total chemical oxygen demand (COD), filtered $\mathrm{COD}, \mathrm{pH}$ value, total solids, total organic solids and ammonium-nitrogen $\left(\mathrm{NH}_{4}\right.$ N) were measured separately for each substrate.

The reliability of the model as a predictor of real plant behaviour is evaluated by comparing real measurements of the plant with simulated results. As measurements, $\mathrm{pH}$ value, biogas production and composition $\left(\mathrm{CH}_{4}, \mathrm{CO}_{2}\right)$, and energy production of the cogeneration units are used. Furthermore, organic acid concentration and VFA/TA (Schoen et al. 2009) are validated periodically by comparison of simulated and real measurement values from laboratory analysis.

Having obtained comparable results (Wolf \& Bongards 20Io), it is assumed that the simulated state of the simulation model is describing the real internal state of the real biogas plant well enough to work with the model as a substitute for the real biogas plant.

\section{The dataset}

As anaerobic digestion is a dynamic process which heavily depends on past states and substrate feeds, past output and substrate feed measurements are used as additional inputs to the static estimation function in the form of a tapped delay line (TDL) with a sampling rate of 6 hours. To reduce the impact of noise, the candidate inputs were selected as moving average estimates of the output measurements taken over intervals of $0.5,1,3,7,14,21$ and 31 days and the substrate feed values over $0.5,1,3$, 7 and 14 days. At each time instant, $t_{\mathrm{i}} \in \mathbf{R}$, these signals are combined with the current values to form vectors $\mathbf{y}_{\text {ext }}\left(t_{\mathrm{i}}\right) \in \mathbf{R}^{m \cdot\left(m_{\mathrm{F}}+1\right)}$ and $\mathbf{u}_{\mathrm{ext}}\left(t_{\mathrm{i}}\right) \in \mathbf{R}^{u \cdot\left(u_{\mathrm{F}}+1\right)}$ for the output and substrate feed measurements, respectively. Here $m_{\mathrm{F}}=7$ (the number of moving average output measurements) and $u_{\mathrm{F}}=5$ (the number of moving average substrate feed measurements).

The identification of the optimal number and combination of moving average inputs in the TDL are investigated in the results section. The final measurement matrix has $m \cdot\left(m_{\mathrm{F}}+1\right)$ columns for the output measurement vector and $u \cdot\left(u_{\mathrm{F}}+1\right)$ columns for the substrate feed measurement vector, i.e. in total $D:=m \cdot\left(m_{\mathrm{F}}+1\right)+u \cdot\left(u_{\mathrm{F}}+1\right)=94$ columns are created. We call this matrix $\mathbf{Y} \in \mathbf{R}^{N \times D}$ :

$\mathbf{Y}:=\left[\begin{array}{c}\mathbf{y}_{\text {ext }}\left(t_{1}\right)^{\mathrm{T}}, \mathbf{u}_{\text {ext }}\left(t_{1}\right)^{\mathrm{T}} \\ \vdots \\ \mathbf{y}_{\text {ext }}\left(t_{N}\right)^{\mathrm{T}}, \mathbf{u}_{\text {ext }}\left(t_{N}\right)^{\mathrm{T}}\end{array}\right]$

where $N \in \mathbf{N}^{+}$is the number of recorded simulation time instances at a sampling time of 6 hours.

In total 75 simulations each lasting 950 days were performed with randomly varying substrate mixtures (defined by $\mathbf{u}$ ), leading to $N=275,850$ samples. The values of each substrate flow were restricted to remain between a lower and an upper bound as can be seen in the left part of Table 1. In the right section of the table the resulting ranges of the measurement values $\mathbf{y}$ are shown.

To be able to apply discriminant analysis and classification methods on the dataset the range for each state vector component $x_{j}$ is clustered into $C=10$ equally distributed classes, $j=1, \ldots, n$. Thus, vectors are generated containing the class labels corresponding to the simulated values of the state vector components $x_{j}$ for each digester, that is, $\vartheta_{1, j} \in\{1, \ldots, C\}^{N}$ and $\vartheta_{2, j} \in\{1, \ldots, C\}^{N}$, $j=1, \ldots, n$.

\section{METHODS}

The complete dataset $\mathbf{Y} \in \mathbf{R}^{N \times D}$ is split into a training dataset $\mathbf{Y}_{\mathrm{T}} \in \mathbf{R}^{N_{\mathrm{T}} \times D}$ and a validation dataset $\mathbf{Y}_{\mathrm{V}} \in \mathbf{R}^{N_{\mathrm{V}} \times D}$ 
(a)

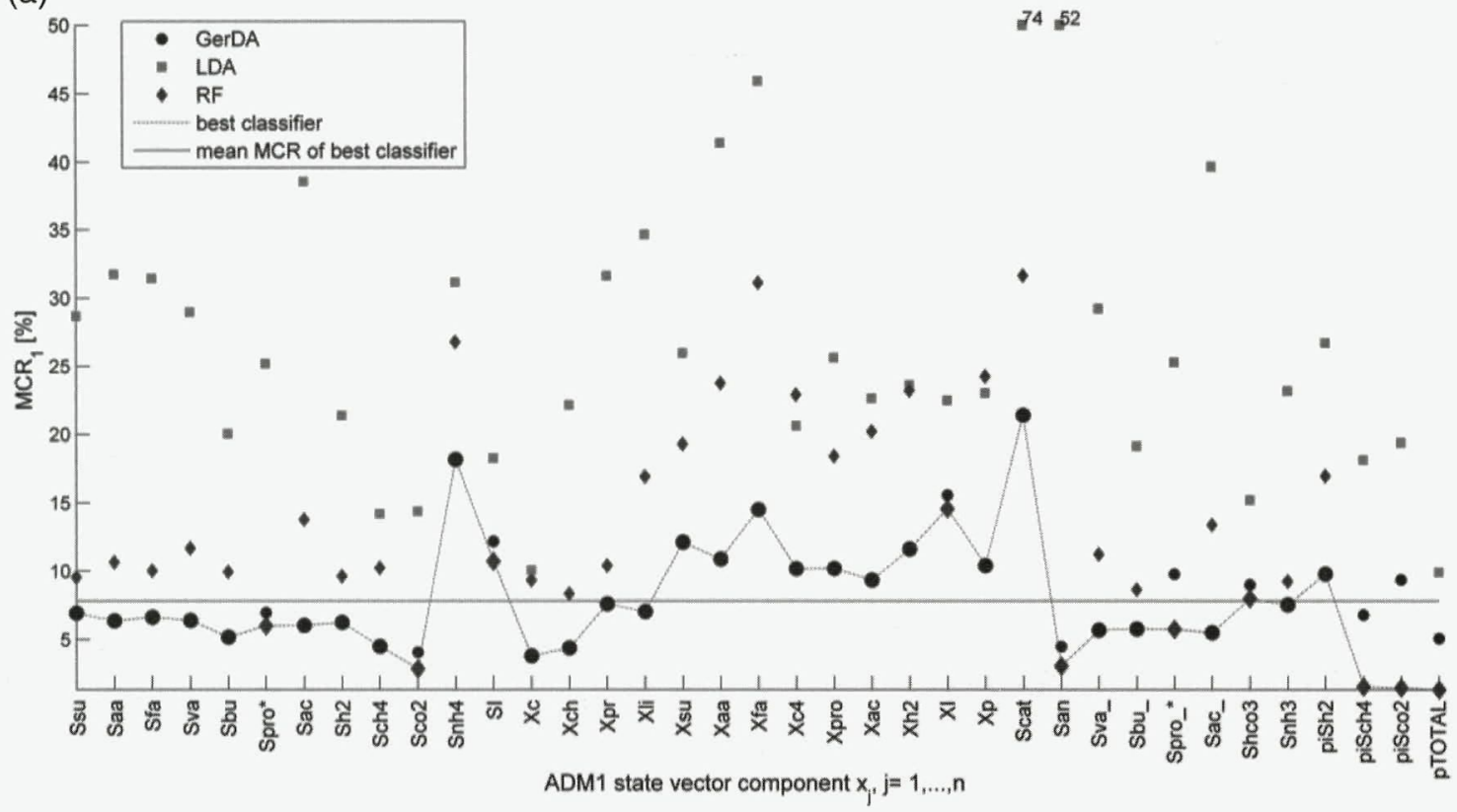

(b)

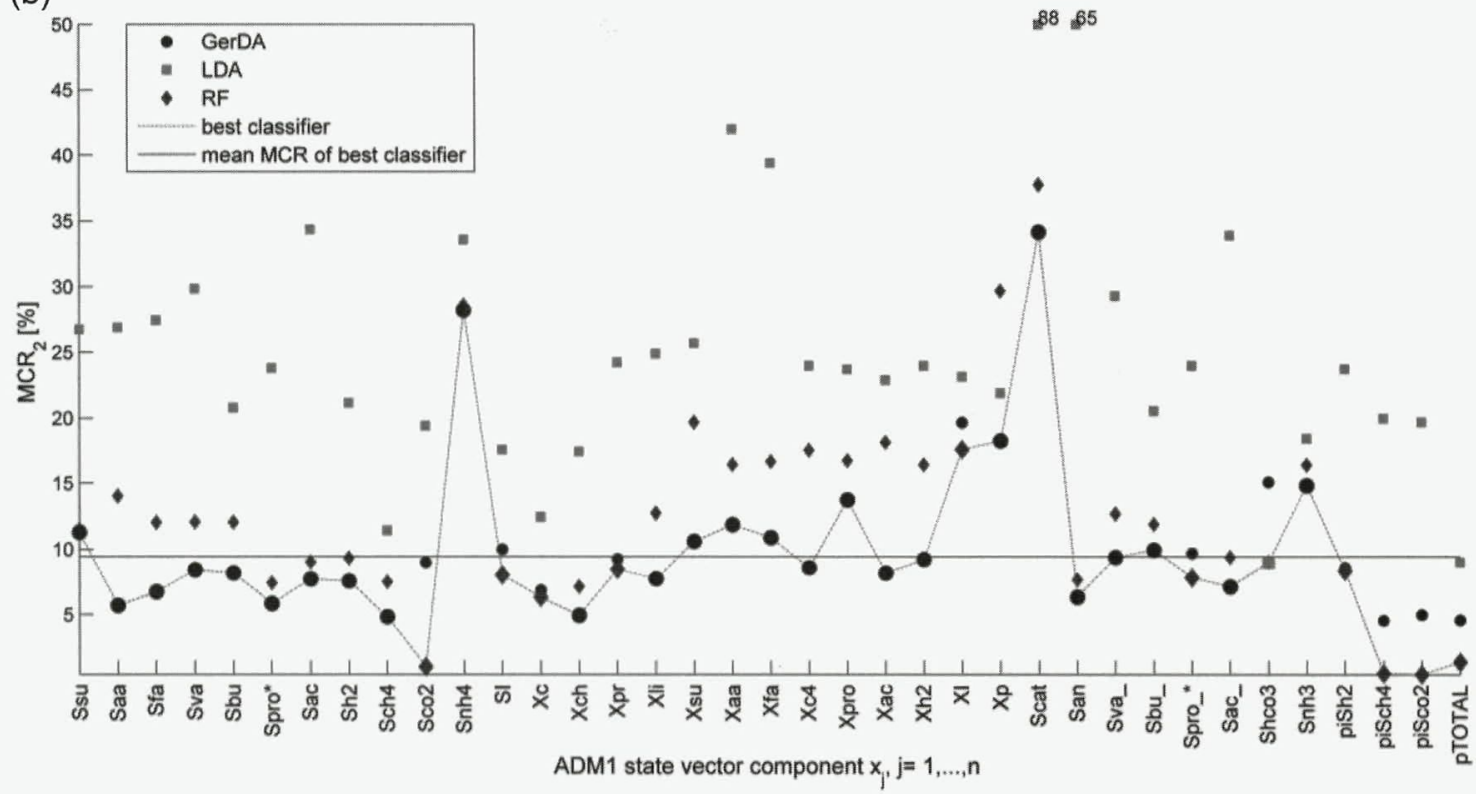

Figure 1 | Comparison of the MCR of the state estimators for each digester using GerDA, LDA and RF: (a) digester 1; (b) digester 2 . The * next to some of the $x$-axis labels signifies that for these state vector components only a $C$ - 1 classification problem was solved, due to insufficient data support for some of the classes. This was addressed by merging such classes with their neighbour class. The best classifier is defined by a combination of the best performing methods for each state vector component.

with $N_{\mathrm{V}}:=N-N_{\mathrm{T}}, N_{\mathrm{T}}<N$. In total five training and five validation datasets are created using five-fold cross-validation. Each training dataset contains the data from 15 selected simulations and thus the validation dataset contains the data from the remaining 60 simulations. In the following, the machine learning methods used are briefly described.

\section{LDA}

Linear Discriminant Analysis searches for a linear transformation $\mathbf{A} \in \mathbf{R}^{d \times D}, d \leq D$, such that the transformed data $\mathbf{Z}=\mathbf{A} \cdot \mathbf{Y}_{\mathrm{T}}^{\mathrm{T}}, \mathbf{Z}:=\left(\mathbf{z}_{1}, \ldots, \mathbf{z}_{N_{\mathrm{T}}}\right) \in \mathbf{R}^{d \times N_{\mathrm{T}}}$, can be linearly separated better than the original feature vectors $\mathbf{Y}_{\mathrm{T}}=\left(\mathbf{y}_{1}, \ldots, \mathbf{y}_{N_{\mathrm{T}}}\right)^{\mathrm{T}}$. The linear transformation $\mathbf{A}$ is 
Table 1 | Range of the measurement matrix Y. Remark: Silo seepage is soaking inside the manure tank, where it has an assumed concentration of $1 \%$, which leads to the bounds below

\begin{tabular}{|c|c|c|c|c|c|c|c|}
\hline Component & Min & Max & Unit & Component & Min & Max & Unit \\
\hline$Q_{\text {maize }}$ & 10.0 & 60.0 & $t / d$ & $\mathrm{pH}_{1}$ & 7.07 & 7.51 & - \\
\hline$Q_{\text {manure }}$ & 20.0 & 110.0 & $\mathrm{~m}^{3} / \mathrm{d}$ & $Q_{\text {biogas }, 1}$ & $2,205.02$ & $9,923.96$ & $\mathrm{~m}^{3} / \mathrm{d}$ \\
\hline$Q_{\text {siloseepage }}$ & 0.15 & 1.15 & $\mathrm{~m}^{3} / \mathrm{d}$ & $V_{\mathrm{CH}_{4}, 1}$ & 45.83 & 55.86 & $\%$ \\
\hline$Q_{\text {grass }}$ & 0.0 & 10.0 & $\mathrm{t} / \mathrm{d}$ & $V_{\mathrm{CO}_{2}, 1}$ & 44.13 & 54.16 & $\%$ \\
\hline \multirow[t]{4}{*}{$Q_{\text {manuresolids }}$} & 0.0 & 10.0 & $\mathrm{t} / \mathrm{d}$ & $\mathrm{pH}_{2}$ & 7.36 & 7.71 & - \\
\hline & & & & $Q_{\text {biogas }, 2}$ & 171.47 & $1,603.26$ & $\mathrm{~m}^{3} / \mathrm{d}$ \\
\hline & & & & $V_{\mathrm{CH}_{4,2}}$ & 57.60 & 67.97 & $\%$ \\
\hline & & & & $V_{\mathrm{CO}_{2}, 2}$ & 32.03 & 42.40 & $\%$ \\
\hline
\end{tabular}

determined by solving an optimization problem maximizing the well-known Fisher discriminant criterion:

$\operatorname{trace}\left\{\mathbf{S}_{\mathrm{T}}^{-1} \cdot \mathbf{S}_{\mathrm{B}}\right\}$

where $\mathbf{S}_{\mathrm{T}}$ is total scatter-matrix and $\mathbf{S}_{\mathrm{B}}$ is the between-class scatter-matrix for the data (Duda et al. 2000). The LDA and a subsequent linear classifier are both implemented in MATLAB ${ }^{\circledR}$. An LDA transformation into a feature space of $d=C-1$ dimensions led to the best subsequent linear classification results.

\section{GerDA}

LDA is a popular pre-processing and visualization tool used in different pattern recognition applications. Unfortunately, LDA followed by linear classification produces high error rates on many real world datasets, because a linear mapping A cannot transform arbitrarily distributed features into independently Gaussian distributed ones. A natural generalization of the classical LDA is to assume a function space $F$ of nonlinear transformations $\mathbf{f}: \mathbf{R}^{D} \rightarrow \mathbf{R}^{d}$ and to still rely on having intrinsic features $\mathbf{Z}:=\mathbf{f}(\mathbf{Y})$ with the same statistical properties as assumed for LDA features. The idea is that a sufficiently large space $F$ potentially contains a nonlinear feature extractor $\mathbf{f}^{*} \in F$ that increases the discriminant criterion (5) compared with a linear extractor $\mathbf{A}$.

GerDA defines a large space $F$ using a Deep Neural Network (DNN), and consequently the nonlinear feature extractor $\mathbf{f}^{*} \in F$ is given by the DNN which is trained with measurements of the data space such that the objective function (5) is maximized. Unfortunately, training a DNN with standard methods, like back-propagation, is known to be challenging due to many local optima in the considered objective function. To efficiently train a large DNN with respect to (5), in Stuhlsatz et al. (2010a, b) a stochastic pre-optimization has been proposed based on greedily layer-wise trained Restricted Boltzmann Machines (Hinton et al. 2006). After layer-wise pre-optimization all weights $\mathbf{W}$ and biases $\mathbf{b}$ of the GerDA-DNN are appropriately initialized. Nevertheless, pre-optimization is suboptimal in maximizing (5), thus a subsequent fine-tuning of the GerDA-DNN is performed using a modified back-propagation of the gradients of (5) with respect to the network parameters. In Stuhlsatz et al. (2010a, b) it is shown that stochastic pre-optimization and subsequent fine-tuning yields very good discriminative features and training time is substantially reduced compared with random initialization of large GerDA-DNNs.

For the extraction of intrinsic features from the raw measurements, we used GerDA with a D-200$100-50-d$ topology, i.e. a five-layer DNN consisting of one input layer with $D$ units, three hidden layers with 200 , 100 respectively 50 units and one output layer with $d$ units resulting in more than 900 million free parameters, with $d=C$. To avoid overfitting the training data, fine-tuning was terminated after the pre-training stage using an earlystopping criterion dependent on the training error. The GerDA-framework is implemented in MATLAB ${ }^{\circledR}$.

\section{Random Forest}

Random Forest is an efficient algorithm for solving complex classification and regression problems based on forming an ensemble of unpruned decision trees (Breiman 200I). Classification is performed by taking the majority vote of an ensemble of classification trees, where each tree is trained on a bootstrapped sample of the original training dataset. In this paper the number of decision trees in the forest is set to 15. The Random Forest algorithm used is from the 
Random Forest implementation for MATLAB ${ }^{\circledR}$ (and Standalone) (Jaiantilal 2010).

\section{RESULTS}

To evaluate the performance of the different methods on the validation datasets the misclassification rate (MCR) is used as a performance measure. This measure is defined as:

$$
\begin{aligned}
& \text { MCR }:=100 \cdot\left(1-\frac{1}{N_{\mathrm{V}}} \cdot \sum_{i=1}^{N_{\mathrm{V}}} 1\left(\mathbf{y}_{i}\right)\right), \\
& 1\left(\mathbf{y}_{i}\right):= \begin{cases}1 & \text { if classified correctly } \\
0 & \text { otherwise }\end{cases}
\end{aligned}
$$

In Figure 1 detailed validation results for each state vector component of both digesters $\left(\mathrm{MCR}_{1}\right.$ and $\left.\mathrm{MCR}_{2}\right)$ are displayed using all three machine learning methods mentioned above. As GerDA is currently implemented using MATLAB $^{\circledR}$ scripts, training for one state vector component takes approximately eighteen hours on a computer with Intel ${ }^{\circledR}$ Xeon ${ }^{\circledR}$ CPU X5550 $(2.67 \mathrm{GHz})$ and 24 GB RAM due to the large amount of data and computational complexity of the algorithm. Nevertheless, as GerDA reveals very good results all components are evaluated using GerDA. If time is an issue then a compromise that can be considered is to only use GerDA to evaluate those components, which are inadequately evaluated using Random Forest or which are identified a priori to be especially important components. Validation however is a matter of seconds for all three methods such that the state estimator is well suited for the use in an online control loop. Currently, the best classifier is a combination of Random Forest, GerDA and LDA, with a mean $\mathrm{MCR}$ of $\mathrm{MCR}_{1}=7.83 \%( \pm 4.43 \%)$ and $\mathrm{MCR}_{2}=$ $9.44 \%( \pm 6.64 \%)$, as can be seen in the figures as well as in Table 2.

Table 2 Performance comparison of the state estimators based on the investigated methods

\begin{tabular}{lll} 
Method & $\begin{array}{l}\mathbf{M C R}_{\mathbf{1}}( \pm \mathbf{~ s t d . ~ d e v . ~}) \\
{[\%]}\end{array}$ & $\begin{array}{l}\mathbf{M C R}_{\mathbf{2}}( \pm \text { std. dev. }) \\
{[\%]}\end{array}$ \\
\hline LDA & $27.20( \pm 12.30)$ & $26.50( \pm 14.49)$ \\
Random Forest & $13.16( \pm 8.05)$ & $12.43( \pm 7.85)$ \\
Random Forest, GerDA \& & $7.83( \pm 4.43)$ & $9.44( \pm 6.64)$ \\
$\quad$ LDA & & \\
\hline
\end{tabular}

From the results, it is apparent that the MCR for Snh4 and Scat is quite high for both digesters and is greater than 30\% for Scat in the second digester. To estimate the expected estimation error the sum over the range of one class and the maximal standard deviation over the rows of the confusion matrix for both components are calculated separately. For Snh4 the range over one class plus the maximal standard deviation is 199 and $229 \mathrm{mg} / \mathrm{L}$ for the first and second digester, respectively. With respect to the measurement range on biogas plants, this range is the same order as the resolution of ion-selective online $\mathrm{NH}_{4}-\mathrm{N}$ measurement sensors, so that in practical terms the expected estimation error is quite small (Hach Lange 20II). For Scat the sum over the range of one class and the maximal standard deviation for the second digester approximately evaluates to $24 \mathrm{mg} / \mathrm{L}$, thus the expected estimation error can be seen as quite small as well (Gerardi 2003).

\section{Results for parameter investigations}

In this section the functional dependency of the results on the number of past measurements in the TDL and the time slots to calculate the average of the past measurements is investigated. In total 32 different configurations of moving horizon filters, by varying amount and window size, are tested using Random Forest. This aspect is done by omitting filters from the original setting. In total for the first digester a mean MCR over the 32 configurations of $13.59 \%( \pm 0.74 \%)$ and for the second digester a mean of $13.16 \%( \pm 1.18 \%)$ was evaluated. The results reveal that the MCR of the state estimator increases more significantly when omitting output filters than when omitting substrate feed filters, when considering only the number of filters and not their window size. This situation is especially true for the second unfed digester, because its behaviour depends only indirectly on the substrate feed. The best result was found by omitting the ' $31 \mathrm{~d}$ ' output filter and the ' $0.5 \mathrm{~d}$ ' substrate feed filter from the original setting, resulting in a mean MCR performance of $12.99 \%( \pm 8.05 \%)$ and $12.67 \%( \pm 8.31 \%)$ for the first and second digester, respectively.

As a further test, the performance of the Random Forest based state estimator was evaluated using noisy measurement data by adding normally distributed random noise to all plant measurements. This showed that the MCR of the state estimator for both digesters increased by a maximum of about $4 \%$ for an increase in the measurement noise of up to $10 \%$. Thus, the state estimator also seems to yield reliable results, when applied to noisy measurements. 


\section{CONCLUSION}

This paper shows that the state of the ADM1 biogas plant model can be predicted using basic $\mathrm{pH}$, biogas production, $\mathrm{CH}_{4}$ and $\mathrm{CO}_{2}$ content and substrate feed measurements that are normally available on commercial biogas plants. Using a combination of Random Forest, GerDA and LDA a good state estimator can be built yielding a MCR of less than $10 \%$. If the simulation model is calibrated for a fullscale biogas plant, as is the case in this paper, the developed state estimator can be implemented online on the plant to gain important information concerning the state of the plant. It is planned to include the developed state estimator in an online NMPC loop for an agricultural biogas plant in the near future.

\section{REFERENCES}

Batstone, D. J., Keller, J., Angelidaki, I., Kalyuzhnyi, S. V., Pavlostathis, S. G., Rozzi, A., Sanders, W. T. M., Siegrist, H. \& Vavilin, V. A. 2002 Anaerobic Digestion Model No.1 (ADM1) (Scientific and Technical Report No. 13). IWA Task Group for Mathematical Modelling of Anaerobic Digestion Processes, IWA Publishing, London.

Bernard, O., Polit, M., Hadj-Sadok, Z., Pengov, M., Dochain, D., Estaben, M. \& Labat, P. 2001 Advanced monitoring and control of anaerobic wastewater treatment plants: software sensors and controllers for an anaerobic digester. Water Science and Technology 43 (7), 175-182.

Breiman, L. 200I Random Forests. Machine Learning 45 (1), 5-32.

Duda, R. O., Hart, P. E. \& Stork, D. G. 2000 Pattern Classification (2nd edn). John Wiley \& Sons, New York.

Etzkorn, C. 2008 Modellierung, messtechnische Analyse und praktische Untersuchung des Fermentationsprozesses an industriellen und landwirtschaftlichen Biogasanlagen (Modelling, Metrological Analysis and Practical Investigation of the Fermentation Process on Industrial and Agricultural Biogas Plants). Diploma Thesis, Cologne University of Applied Sciences, Gummersbach.

Directive 2009/28/EC of the European Parliament and of the Council of 23 April 2009 on the promotion of the use of energy from renewable sources and amending and subsequently repealing Directives 2001/77/EC and 2003/30/ EC, European Parliament 2009.

Fortuna, L., Graziani, S., Rizzo, A. \& Xibilia, M. G. 2007 Soft Sensors for Monitoring and Control of Industrial Processes. Springer Verlag.

Gaida, D., Wolf, C., Bongards, M. \& Bäck, T. 2опа MATLAB toolbox for biogas plant modelling and optimization. In: Progress in Biogas II - Biogas Production from Agricultural Biomass and Organic Residues. FnBB e.V. (Fördergesellschaft für nachhaltige Biogas - undBioenergienutzung e.V.). German
Society for sustainable Biogas and Bioenergy Utilization, Stuttgart, Vol. 2, pp. 67-70.

Gaida, D., Sousa Brito, A. L., Wolf, C., Bäck, T., Bongards, M. \& McLoone, S. 2omb Optimal control of biogas plants using nonlinear MPC. In: ISSC 2011, vol. 1, pp. 219-224.

Gerardi, M. H. 2003 The Microbiology of Anaerobic Digesters (1st ed.). Wastewater Microbiology Series. Wiley-Interscience, Hoboken, NJ.

Hach Lange 20I AN-ISE sc: Combination sensor for ammonium and nitrate: Data Sheet. Data Sheet. Retrieved from http://www.fr.hach-lange.be/shop/action_q/download\% 3Bdocument/DOK_ID/14791228/type/pdf/lkz/BE/spkz/ nl/TOKEN/hrBDyMZFRi6xL-h8VgF5PfN1BAM/M/ ZWZYLQ.

Hinton, G. E., Osindero, S. \& The, Y. W. 2006 A fast learning algorithm for deep belief nets. Neural Computation 18 (7), $1527-1554$.

Jaiantilal, A. 20Io randomforest-matlab (Version 0.02). Retrieved from http://code.google.com/p/randomforest-matlab/.

Koch, K., Lübken, M., Gehring, T., Wichern, M. \& Horn, H. 2010 Biogas from grass silage - measurements and modeling with ADM1. Bioresource Technology 101 (21), 8158-8165.

Lübken, M., Wichern, M., Schlattmann, M., Gronauer, A. \& Horn, H. 2007 Modelling the energy balance of an anaerobic digester fed with cattle manure and renewable energy crops. Water Research 41 (18), 4085-4096.

Page, D. I., Hickey, K. L., Narula, R., Main, A. L. \& Grimberg, S. J. 2008 Modeling anaerobic digestion of dairy manure using the IWA Anaerobic Digestion Model No. 1 (ADM1). Water Science and Technology 58 (3), 689-695.

Rawlings, J. B. \& Bakshi, B. R. 2006 Particle filtering and moving horizon estimation. Computers and Chemical Engineering 30 (10-12), 1529-1541.

Schmitz 2004 Landwirtschaftskammer NRW (Chamber of Agriculture for North Rhine Westphalia).

Schoen, M. A., Sperl, D., Gadermaier, M., Goberna, M., FrankeWhittle, I., Insam, H., Ablinger, J. \& Wett, B. 2009 Population dynamics at digester overload conditions. Bioresource Technology 100 (23), 5648-5655.

Stuhlsatz, A., Lippel, J. \& Zielke, T. 2oroa Discriminative feature extraction with deep neural networks. In: Proceedings of the 2010 International Joint Conference on Neural Networks (IJCNN), Barcelona, Spain.

Stuhlsatz, A., Lippel, J. \& Zielke, T. 2orob Feature extraction for simple classification. In: Proceedings of the International Conference on Pattern Recognition (ICPR). Istanbul, Turkey.

Wichern, M., Lübken, M., Koch, K., Gehring, T., Horn, H., Fischer, K., Schlattmann, M. \& Gronauer, A. 2007 Eignung des Anaerobic Digestion Model No. 1 (ADM 1) zur Prozesssteuerung landwirtschaftlicher Biogasanlagen (Applicability of the Anaerobic Digestion Model No. 1 (ADM 1) for process control of agricultural biogas plants). Gülzower Fachgespräche - Messen, Steuern, Regeln bei der Biogaserzeugung 27, 172-194.

Wichern, M., Gehring, T., Fischer, K., Andrade, D., Lübken, M., Koch, K., Gronauer, A. \& Horn, H. 2009 Monofermentation 
of grass silage under mesophilic conditions: measurements and mathematical modeling with ADM 1. Bioresource Technology 100, 1675-1681.

Wiese, J. \& König, R. 2009 From a black-box to a glass-box system: the attempt towards a plant-wide automation concept for full-scale biogas plants. In: Water Science and
Technology (H. Kroiss, ed.). London, IWA Publishing, pp. 321-327.

Wolf, C. \& Bongards, M. 2010 Modellbasierte Prozessoptimierung von Biogasanlagen (MOBIO) (Model-based process optimisation of biogas plants). Final report. Cologne University of Applied Sciences \& PlanET GmbH, Gummersbach, Germany.

First received 15 February 2012; accepted in revised form 16 April 2012 
Copyright of Water Science \& Technology is the property of IWA Publishing and its content may not be copied or emailed to multiple sites or posted to a listserv without the copyright holder's express written permission. However, users may print, download, or email articles for individual use. 